\title{
Control of magnetic anisotropy and magnetic patterning of perpendicular Co/Pt multilayers by laser irradiation
}

\author{
C. Schuppler, A. Habenicht, and I. L. Guhr \\ Fachbereich Physik, Universität Konstanz, Universitätsstrasse 10, 78457 Konstanz, Germany \\ M. Maret \\ Laboratoire de Thermodynamique et Physico-Chimie Métallurgiques, ENSEEG BP 75, \\ 38402 Saint Martin d'Heres, France \\ P. Leiderer, J. Boneberg, and M. Albrecht ${ }^{\mathrm{a})}$ \\ Fachbereich Physik, Universität Konstanz, Universitätsstrasse 10, 78457 Konstanz, Germany
}

(Received 16 August 2005; accepted 5 December 2005; published online 5 January 2006)

\begin{abstract}
We report an approach to altering the magnetic properties of (111) textured $\mathrm{Co} / \mathrm{Pt}$ multilayer films grown on sapphire (0001) substrates in a controlled way using single-pulse laser irradiation. The as-grown films reveal a strong perpendicular magnetic anisotropy induced by interfacial anisotropy. We show that laser irradiation can chemically mix the multilayer structure particularly at the interfaces, hence reducing the perpendicular magnetic anisotropy and coercivity in a controlled manner depending on laser fluence. As a result, perpendicular films can also be magnetically patterned into hard and soft magnetic regions using a regular two-dimensional lattice of polystyrene particles acting as an array of microlenses.
\end{abstract}

Many magnetic multilayer systems such as $\mathrm{Co} / \mathrm{Pt}$ exhibit large coercivities and strong perpendicular magnetic anisotropies due to the contribution of the interfaces. ${ }^{1}$ As the magnitude of this so-called interfacial anisotropy is known to depend sensitively on the sharpness of the interface, ${ }^{2}$ it is not surprising that the perpendicular magnetic anisotropy could be reduced by effects that cause interfacial mixing. In this regard, various techniques ${ }^{3-6}$ are well established for systematically tailoring the magnetic properties, however, limited mainly to ion-beam irradiation. For the latter, it is known that the coercivity of $\mathrm{Co} / \mathrm{Pt}$ multilayers falls with increasing ion dose, and this is assumed to be a result of collision-induced mixing of the different atoms, reducing the interfacial anisotropy. ${ }^{6-8}$ Based on these results, magnetic patterning of perpendicular multilayer films has been exploited using focused ion beams ${ }^{9}$ or ion irradiation through a mask ${ }^{6-8,10}$ to create local modifications of film properties. Another approach to modify material properties can be achieved by laser irradiation as studied in spin-valve systems, ${ }^{11}$ which also lead to intermixing of the interfaces in multilayer film structures. This technique can be extended to magnetic patterning that can be achieved by projection of the laser light via a mask, by the interference of laser beams or by employing a direct-contact mask such as a two-dimensional array of spherical particles. ${ }^{12}$ For the latter, the incident laser beam can pass through the particle mask and is focused onto the sample by the particles, which act as a microlensing system, thereby enhancing the laser intensity underneath the particles. $^{13,14}$

We report in this letter the controlled modification of magnetic properties of perpendicular $\mathrm{Co} / \mathrm{Pt}$ multilayer films induced by laser irradiation. The interplay between magnetic and structural properties was monitored by x-ray diffraction

${ }^{a}$ Author to whom correspondence should be addressed; electronic mail: manfred.albrecht@uni-konstanz.de
(XRD), atomic and magnetic force microscopy (AFM/MFM) and superconducting quantum interference device (SQUID) magnetometry. As a result of the laser-controllable anisotropy, magnetic patterning via focusing light by spherical particle arrays was demonstrated.

$\mathrm{Co} / \mathrm{Pt}$ multilayer films were deposited by evaporation of $\mathrm{Co}$ and $\mathrm{Pt}$ from two e-beam sources at room temperature on sapphire (0001) substrates. Circular samples with a diameter of $1.3 \mathrm{~mm}$ were fabricated using a shadow mask. In our studies, multilayers consisting of a $[\mathrm{Co}(0.3 \mathrm{~nm}) / \mathrm{Pt}(0.8 \mathrm{~nm})]_{12}$ stack deposited on a $1 \mathrm{~nm} \mathrm{Cr} / 3 \mathrm{~nm}$ Pt seed layer stack were used and covered by an additional 1-nm-thick Pt layer to prevent oxidation. These films exhibit strong perpendicular anisotropy of about $0.4 \mathrm{MJ} / \mathrm{m}^{3}$ consistent with values reported in literature. ${ }^{15}$

Laser irradiation was performed by using a frequencydoubled Nd:yttrium-aluminum-garnet laser system $(\lambda$ $=532 \mathrm{~nm}$ ) applying a single laser pulse at fixed full width at half maximum (FWHM) of about $10 \mathrm{~ns}$. The FWHM of the beam spot was about $3.5 \mathrm{~mm}$, which provides an almost homogeneous intensity profile across the sample. The laser fluence at the surface was varied between 90 and $230 \mathrm{~mJ} / \mathrm{cm}^{2}$, The angle of incidence of the beam was $45^{\circ}$ with respect to the surface normal of the film. A crude estimate of the expected maximum temperature suggests melting to start around $180 \mathrm{~mJ} / \mathrm{cm}^{2}$.

Before laser irradiation, each sample was characterized by SQUID magnetometry, measuring hysteresis loops at room temperature with the field applied perpendicular and parallel to the film plane. The resulting loops after laser irradiation were analyzed to yield effective magnetic anisotropy $\left(K_{\text {eff }}\right)$, coercivity $\left(H_{c}\right)$, and saturation magnetization $\left(M_{s}\right)$ and were compared to the initial values. Figure 1 shows the evolution of the $M-H$ loops with increasing laser fluence. The loops reveal a pronounced softening of the films with increasing fluence until the perpendicular anisotropy falls al- 


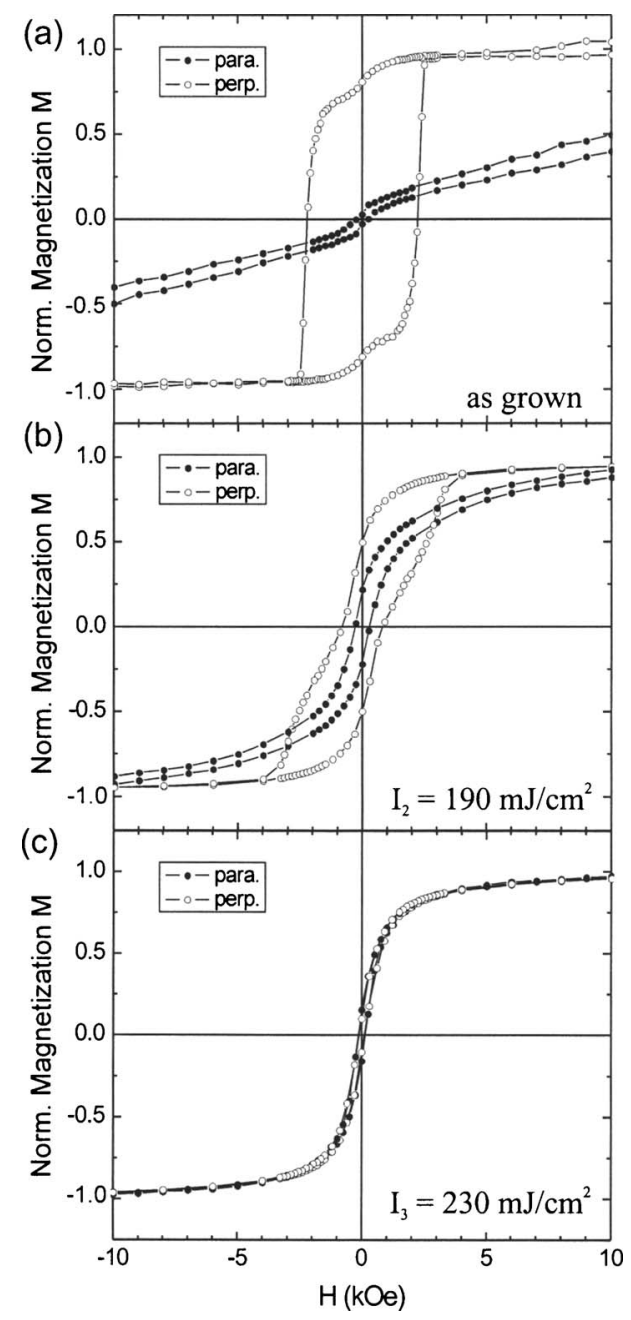

FIG. 1. SQUID $M-H$ hysteresis loops showing the evolution of the loops after laser irradiation at various fluences. The loops were measured in a magnetic field of up to $\pm 50 \mathrm{kOe}$, and the field was applied perpendicular (open circles) and parallel (filled circles) to the film plane.

most to zero and the magnetization starts rotating into the film plane. The values of the effective magnetic anisotropy are given in Fig. 2(a) as a function of applied laser fluence. These results show the same trends as have been reported previously using ion irradiation even though the underlying mechanism of interface mixing is quite different. Interestingly, at the same time, the saturation magnetization of the samples increases substantially with increasing fluence as shown in Fig. 2(b), as can be explained by induced polarization of $\mathrm{Pt}$ atoms surrounded now by $\mathrm{Co}$ atoms. However, the strong increase in saturation magnetization of about $65 \%$ obtained for the highest fluence is still under investigation and could be related to the formation of an ordered Co-Pt alloy.

In order to investigate in more detail the structural changes of the multilayer films after irradiation, XRD was performed at small and high angles with a four-circle Huber diffractometer using $\mathrm{Cu} K \alpha_{1}$ radiation. High-angle XRD investigations on the as-grown sample show on the left side of the strong (006) sapphire reflection a broad peak coming from the multilayer stacking. As evident from Fig. 3(a), for fluences up to $160 \mathrm{~mJ} / \mathrm{cm}^{2}$ this peak shifts to larger angles corresponding to a decrease of the interatomic distance between (111) planes from 2.215 to $2.20 \AA$ and then for larger fluences increases strongly in intensity. This drastic change in intensity can be attributed to an almost complete mixing
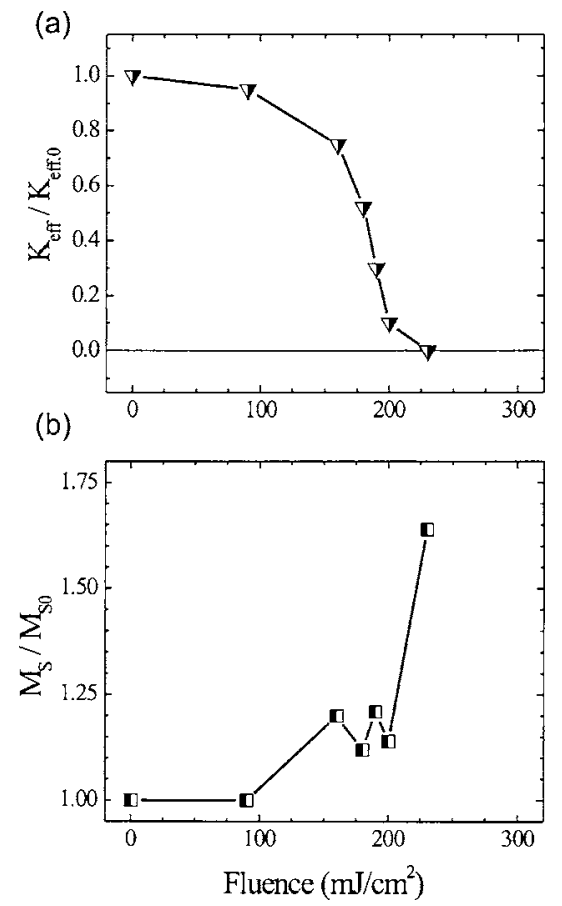

FIG. 2. (a) Ratio of the effective magnetic anisotropy constant and (b) of the saturation magnetization after and before irradiation as a function of applied laser fluence.

between the thin Co layers and Pt layers leading to an homogeneous alloy film. This is supported by the FWHM of the (111) peak, which gives a coherence length equal to 16.6 $\mathrm{nm}$ for the multilayer irradiated under a fluence of $190 \mathrm{~mJ} / \mathrm{cm}^{2}$. Figure 3(b) shows small-angle XRD scans revealing Kiessig fringes connected to the total film thickness and the first multilayer peak located around $8^{\circ}$. This
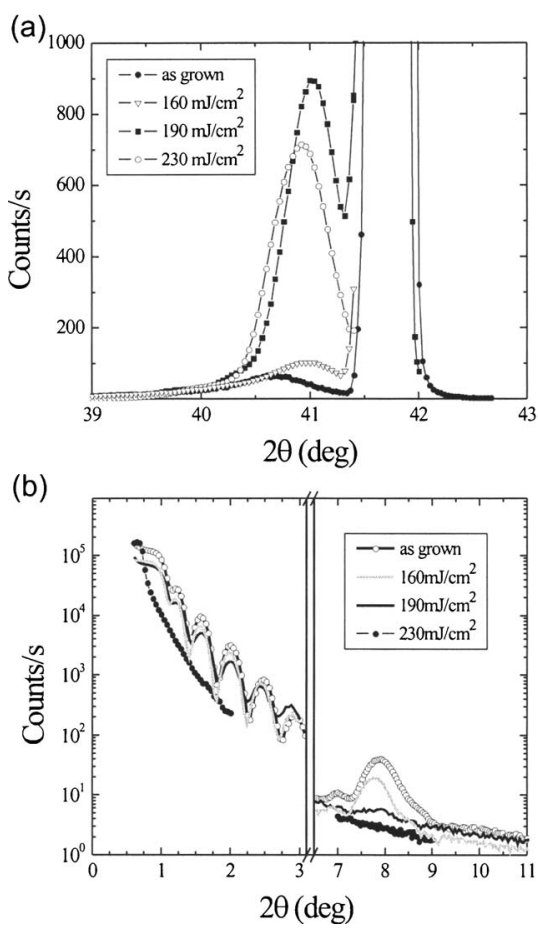

(c)

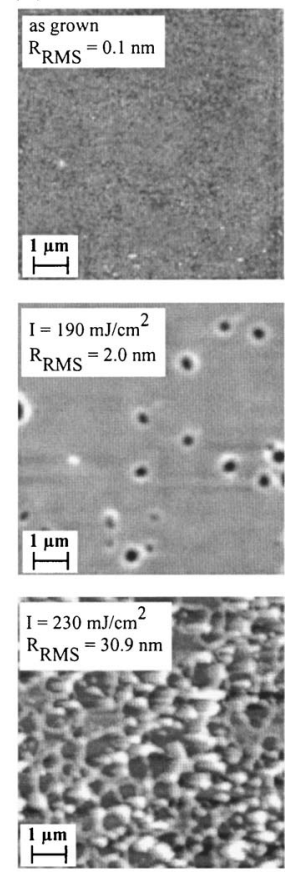

FIG. 3. XRD $\theta-2 \theta$ scans at (a) high and (b) low angles revealing the evolution of the structural properties induced by laser irradiation. (c) AFM images showing the corresponding surface morphology of the films with values of the route-mean-square (rms) roughness. 


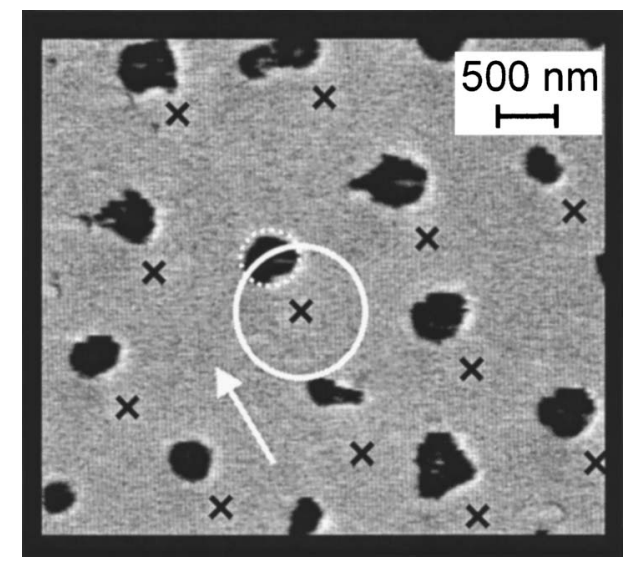

FIG. 4. MFM image after laser irradiation employing a $1.2 \mu \mathrm{m}$ particle monolayer on a Co/Pt multilayer film. The crosses in the image mark the initial PS particle positions as obtained from corresponding AFM imaging while the dotted circle shows the irradiated area. The arrow indicates the beam direction, which is tilted by $45^{\circ}$ to the surface normal.

multilayer peak gradually weakens with laser fluence and disappears at a fluence of $230 \mathrm{~mJ} / \mathrm{cm}^{2}$ demonstrating the occurrence of chemical mixing at the interfaces. Along with the intermixing, a drastic change in surface morphology of the films is indicated by the vanishing Kiessig fringes. Simulations of the small-angle reflectivity curve ${ }^{16}$ for the as-grown sample lead to average values of the Co and Pt layers thicknesses equal to 2.1 and $9.1 \AA$ with corresponding rms roughnesses of 5.4 and $4.2 \AA$. The best fits were obtained using a linear increase of the Pt layer roughness of 50\% (from the bottom to the top of the multilayer) and slightly larger for the Co layers. The changes observed after an irradiation of $190 \mathrm{~mJ} / \mathrm{cm}^{2}$ were reproduced by changing the scattering density contrast between the two layers (i.e., changing the scattering density from 4 to $3.8 \mathrm{e} / \AA^{3}$ for the Pt layers and from 2.4 to $3 \mathrm{e} / \AA^{3}$ for the Co layers), thus confirming strong intermixing. To characterize the morphology of the irradiated films, AFM studies were carried out, and the corresponding images are presented in Fig. 3(c). While the as-grown film shows a rather flat film topography, the film irradiated at a fluence of $190 \mathrm{~mJ} / \mathrm{cm}^{2}$ reveals individual holes, turning to a crater-like film topography at a fluence of $230 \mathrm{~mJ} / \mathrm{cm}^{2}$. Both observations indicate that the film material undergoes a very fast melting and cooling process accompanied by dewetting processes, which are well known from the literature. ${ }^{17}$

Magnetic patterning was performed employing a colloidal monolayer of polystyrene (PS) particles with a diameter of $1.2 \mu \mathrm{m}$ and using a single $532 \mathrm{~nm}$ laser pulse with a fluence of $80 \mathrm{~mJ} / \mathrm{cm}^{2}$. The monolayers were formed directly on the $\mathrm{Co} / \mathrm{Pt}$ multilayer films. ${ }^{18,19}$ As long as the laser wavelength is smaller than or comparable to the particle diameter, the spherical particles act as microlenses that focus the incident laser irradiation onto the film sample. Looking back on the results of the films, we expected to induce local magnetic changes without changes of surface morphology. From AFM imaging of the patterned area of the film, the footprint of the initial positions of the PS particles, revealing a quasi hexagonal lattice structure, are still visible on the surface even after the particles are removed. ${ }^{19}$ However, no further topographic features were observed in the AFM image, while the corresponding MFM image presented in Fig. 4 showed a strong magnetic contrast. In this picture, the initial positions of the particles are indicated by the crosses, the size of one of the particles is given by the full white circle, while the focus of this particle is indicated by the dotted circle. As the MFM picture demonstrates, the laser fluence locally transforms the magnetically hard $\mathrm{Co} / \mathrm{Pt}$ multilayer film into a magnetically much softer material. The magnetic pattern is formed by the demagnitization fields generated by the magnetically hard environment after saturation, thereby reversing the magnetization of the soft magnetic areas. It is apparent from Fig. 4 that the magnetic structures, look quite different in shape and magnetic contrast mainly due to differences in particle size, leading to drastic local variations in intensity of the scattered laser light.

In conclusion, laser irradiation has been shown to alter the magnetic properties of thin $\mathrm{Co} / \mathrm{Pt}$ multilayer films in a controlled way. Our results indicate that the fall in perpendicular magnetic anisotropy and rise in saturation magnetization are associated with intermixing of the interfaces. Magnetic patterning was demonstrated using laser irradiation employing two-dimensional particle arrays over areas of a square millimeter and further investigations are in progress to study the physical limitations of this technique.

Financial support by the Deutsche Forschungsgemeinschaft through the SFB 513 and the Emmy-Noether program is gratefully acknowledged. The authors would like to thank Professors W. Evenson and G. Schatz for fruitful discussions.

${ }^{1}$ I. K. Schuller, S. Kim, and C. Leighton, J. Magn. Magn. Mater. 200, 571 (1999).

${ }^{2}$ J. M. MacLaren and R. H. Victora, Appl. Phys. Lett. 66, 3377 (1995).

${ }^{3}$ P. F. Carcia, S. I. Shah, and V. Zeper, Appl. Phys. Lett. 56, 2345 (1990).

${ }^{4}$ M. Van Rossum, M.-A. Nicolet, and C. H. Wilts, J. Appl. Phys. 56, 1032 (1984).

${ }^{5}$ C. Tosello, L. M. Gratton, G. Principi, A. Gupta, and R. Gupta, Surf. Coat. Technol. 84, 338 (1996).

${ }^{6}$ C. Chappert, H. Bernas, J. Ferré, V. Kottler, J.-P. Jamet, Y. Chen, E. Cambril, T. Devolder, F. Rousseaux, V. Mathet, and H. Launois, Science 280, 1919 (1998).

${ }^{7}$ B. D. Terris, L. Folks, D. Weller, J. E. E. Baglin, A. J. Kellock, H. Rothuizen, and P. Vettiger, Appl. Phys. Lett. 75, 403 (1999).

${ }^{8}$ D. Weller, J. E. E. Baglin, A. J. Kellock, K. A. Hannibal, M. F. Toney, S. Lang, L. Folks, M. E. Best, and B. D. Terris, J. Appl. Phys. 87, 5768 (2000).

${ }^{9}$ T. Aign, P. Meyer, S. Lemerle, J. P. Jamet, J. Ferré, V. Mathet, C. Chappert, J. Gierak, C. Vieu, F. Rousseaux, H. Launois, and H. Bernas, Phys. Rev. Lett. 81, 5656 (1998).

${ }^{10}$ T. Devolder, C. Chappert, V. Mathet, H. Bernas, Y. Y. Chen, H. Ravera, J. P. Jamet, and J. Ferré, J. Appl. Phys. 87, 8671 (2000).

${ }^{11}$ Š. Luby, E Majková, M. Spasova, M. Jergel, R. Senderák, E. d'Anna, A. Luches, M. Martino, M. Brunel, and I. M. Dmitrenko, Thin Solid Films 312, 15 (1998).

${ }^{12}$ N. I. Polushkin, Appl. Phys. Lett. 86, 132502 (2005).

${ }^{13}$ H.-J. Münzer, M. Mosbacher, M. Bertsch, J. Zimmermann, P. Leiderer, and J. Boneberg, J. Microsc. 202, 129 (2001).

${ }^{14}$ P. Leiderer, C. Bartels, J. König-Birk, M. Mosbacher, and J. Boneberg, Appl. Phys. Lett. 85, 5270 (2004).

${ }^{15}$ C.-J. Lin, G. L. Gorman, C. H. Lee, R. F. C. Farrow, E. E. Marinero, H. V. Do, H. Notarys, and C. J. Chien, J. Magn. Magn. Mater. 93, 194 (1991).

${ }^{16}$ S. I. Zabinsky, J. J. Rehr, A. Ankudinov, R. C. Albers, and M. J. Eller, Phys. Rev. B 52, 2995 (1995).

${ }^{17}$ J. Bischof, D. Scherer, S. Herminghaus, and P. Leiderer, Phys. Rev. Lett. 77, 1536 (1996).

${ }^{18}$ U. C. Fischer and H. P. Zingsheim, J. Vac. Sci. Technol. 19, 881 (1981).

${ }^{19}$ F. Burmeister, W. Badowsky, T. Braun, S. Wieprich, J. Boneberg, and P. Leiderer, Appl. Surf. Sci. 144-145, 461 (1999). 